\title{
Induction of tumor cell apoptosis in human glioblastoma cell lines by cationic peptides
}

\begin{abstract}
Background: Glioblastoma is very aggressive polymorphic brain tumor that is often chemo- and radio-resistant. This feature is related with glioma stem-like cells as well as with non-stem changeable cells from glioblastoma's cell population. Search for molecular targets to overcome such resistance and to improve the effectiveness of therapy is one of the major challenges in applied molecular oncology.

Objective: The aim of this study is to analyze a selective cytotoxicity of two cationic peptides (CPs) detected on two stable primary glioblastoma cell lines Glb0Sh and Glb-17. Induction of apoptosis in the cultures of recurrent glioblastoma cells and lack of cytotoxicity in normal cells was revealed by MTT assay, immunocytochemistry visualization of tumor cells after incubation with fluorescently labeled CP, RT PCR and western blotting.

Results: We firstly confirm that chaperone proteins nucleolin/NCL and nucleophosmin/ NPM serve as cell molecular targets for CPs under study which has shown high selective cytotoxicity in two stable glioblastoma cell lines. These CPs are of interest for in vivo experiments as a promising anticancer agents.
\end{abstract}

Keywords: primary glioblastoma cell lines, cationic peptides (CPs), selective tumor cell cytotoxicity, nucleolin, nucleophosmin specific expression, apoptosis
Volume I2 Issue 5 - 202I

\author{
Anna A Lushnikova,' Anastasia $\vee$ Onyan, ${ }^{3}$ \\ Alexander V Kostarev, ${ }^{2}$ Ekaterina Yu \\ Rybalkina, ${ }^{3}$ Ksenia V Kohzikhova, ${ }^{4}$ Sergey $M$ \\ Andreev $^{5}$ \\ 'Leader researcher, Head of Oncogenomics Llaboratory, NN \\ Blokhin Cancer Research Center, Russia \\ 2Post Graduate Student, Department of Physics, MV Lomonosov \\ Moscow State University, Russia \\ ${ }^{3}$ Researcher, Oncogenomics Lab, NN Blokhin Cancer Research \\ Center, Russia \\ ${ }^{4}$ Researcher, State Sci Center "Immunology Institute", Russia \\ ${ }^{5}$ Head of Laboratory, State Sci. Center "Immunology Institute", \\ Russia
}

Correspondence: Dr. Anna A Lushnikova, NN Blokhin Cancer Research Center, I I 5478 Kashirskoye shosse-24, Moscow, Russia, Email LAN2I@yandex.ru

Received: September 28, 202I | Published: October 20, 202 I

\section{Introduction}

The most human malignant tumors with late manifestation and metastases are resistant to standard therapy. Such neoplasms include glioblastomas, melanomas, pancreatic cancer, renal cancer and a number of other malignant tumors. The development of molecular targeted drugs with high activity against aggressive chemoresistant tumors, with low toxicity to normal cells is a challenge in modern oncology.

Earlier, we investigated the cytotoxicity of a number of cationic peptides (PCs) with a molecular weight of $1-2.5 \mathrm{kDa}$ and a charge from +4 to +16 , which have shown selective toxicity against a number of tumor cell lines and corresponding xenographts. ${ }^{1,3}$ These PCs penetrate well into cells and they are resistant to intracellular degradation. Now PCs are used for intracellular transport of biomolecules as complexes $\mathrm{CP}$-aptamer or CP-drug, CP-microRNA, etc. ${ }^{1}$ Nucleolin is expressed on the surface of tumor cells and interacts with various ligands, including CPs. Nucleolin and nucleophosmin are also highly expressed in the tumor cells themselves and in the cell nuclei. These poly-functional proteins play an important role in the regulation of important cellular processes, including transcription. Translation, ribosome biogenesis, cell proliferation, intracellular transport and signaling, apoptosis, cell differentiation, epithelial mesenchymal transition, etc. ${ }^{2-4}$

Recently, cell surface nucleolin has been considered as a cellular target for the delivery of antitumor drugs in vivo and for tissuespecific inhibition of tumor progression in brain, lung, stomach, hepatic area, and other localizations. ${ }^{5,6}$ Moreover, cell surface NCL over expression in tumor cells with stem-like phenotype enables targeted intracellular drug delivery. ${ }^{7}$ It has been shown, that N6L-the synthetic antagonist of cell surface nucleolin in combination with the plant toxin saporin induces the death of glioblastoma cells in vitro at a concentration 40 times less $(0.1 \mathrm{nM})$ than free N6L. The volume of orthotropic xerographs of human glioblastoma has decreased after weekly administration of $\mathrm{N} 6 \mathrm{~L}, 0.75 \mu / \mathrm{ml} ; 0.5 \mathrm{mg} / \mathrm{kg}$ per mouse during 4 weeks. $^{7}$ Here, we have studied the cytotoxicity of two dendritic CPs as potential antitumor agents for human brain cancer using two glioblastoma cell lines.

\section{Materials and methods}

Brain glioblastoma cell lines obtained from surgical biopsies were studied: (1) Glb-Sh line was generated from male patient with glioblastoma multiform, diagnosis manifestation was at 2011, fatal outcome was a year later; (2) Glb-17 line was generated from male patient with low-grade glioblastoma, the diagnosis manifestation was in 2013, surgical intervention - in 2014, the disease outcome was not followed.

The cells were incubated in RPMI medium supplied with $10 \%$ embryonic bovine serum. Cytotoxicity of CPs was analyzed in standard MTT test after 3 days of incubation with dilutions from 0.5 to $4 \mu \mathrm{g} / \mathrm{ml}$. A transferable line of human skin fibroblasts H1036 was used as a control. In addition, the cells were passioned into microcells on the slide (Cell imaging slide, Eppendorf, Germany); a day later, cells were incubated for 2-6 hours CP, fluorescently labeled by cyanine $\mathrm{Cy} 5-\mathrm{NC} 811(1 \mathrm{mg} / \mathrm{ml})$ to detect $\mathrm{CP}$ transport. Cell apoptosis was revealed using staining with Hoechst 33342, DAPI, PI, annexin-fluorescein (Ann-Cy3/6-CFDA), Sigma-Aldrich. For immunohistochemical staining of glioblastoma cells we used commercial monoclonal antibodies to the nuclear fractions of NCL and NPM (Ye, GEP, Life Technology, USA) and visualization by Gel Sustain/Abcam Immunocytochem Detection System (DIAEM, Russia).using Image Analysis program MatLab 6.0. For western bloting, monoclonal antibodies to NCL/NPM/P53 were used according to the manufacturer's recommendations (Abcam/DIAEM) (Table 1). 
Table I Some physical and chemical properties of the peptides

\begin{tabular}{|c|c|c|c|c|}
\hline$\#$ & Formula & Molecular weight/charge & Effective concentration $(\mu \mathrm{g} / \mathrm{ml})$ & $\begin{array}{l}\text { Proportion of alive cells in Glb } \\
\text { Sh/Glb-I } 7 \text { (min-max) in MTT } \\
\text { tests (\%) }\end{array}$ \\
\hline I & AM-2/Mir-KRPARPAR-NH ${ }_{2}$ & $139 \mid / 4+$ & $\geq 0.50$ & $6-9 / 13-25$ \\
\hline 2 & 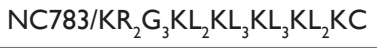 & $25050 / 9+$ & $\geq 0.25$ & $8-12 / 9-15$ \\
\hline
\end{tabular}

For western blotting, the protein concentration was determined by the Bradford method (BioRad) and fluorescently labeled monoclonal antibodies to NCL, NPM, p53 and b-actin conjugated with horseradish peroxidase and the first mouse IgG antibodies (Sigma, USA) were used after protein immunoprecipitation, 7/5\% SDS-PAGE electrophoresis and transfer to a nitrocellulose filter. Two problems had to be solved before molecular docking: 1) the sites of binding between the target protein and peptide are not exactly known. Sitemap program Maestro 11, has not shown reasonable binding areas, apparently, due to the fact, that there no large hydrophobic holes on the protein surface. So, we used a maximal docking box size of $36 \AA$ and this area included the entire protein. Thus, one can calculate the potential grid throughout the protein using glide; 2) the peptides were too large for glide, so we decided to divide their molecules into peptide fragments enriched with charged groups. The fragments of different sizes were quite large, so the result of the docking was meaningful. The ligand preparation procedure has also included protonation, $\mathrm{pH}=7.0$. Moreover, we have done 4 conformers for each ligand . The docking was performed by glide. $^{8}$

\section{Results}

Nucleolin/NCL and nucleophosmin/NPM are highly expressed in the most of solid tumors and represent novel therapeutic target. Nucleolin is expressed at the plasma membrane of tumor cells and it is considered as a marker for different cancers, including glioblastoma. Targeting cell surface NCL might be a new therapeutic strategy for glioblastoma treatment. To explore this possibility, the effect of an antagonist of NCL - the multivalent pseudopeptide N6L, using primary culture of human glioblastoma cells was studied. Cell cycle analysis indicated that N6L-induced growth reduction was due to a block of the G1/S transition with down-regulation of the expression of cyclin D1 and B2. Moreover, the level of autophagy markers p62 and LC3II was enhanced after N6L treatment. In addition, N6L-treatment of mice bearing tumor decreased in vivo tumor growth in orthotopic brain tumor model and increase mice survival. ${ }^{9}$ The results obtained by these authors, indicated an anti-proliferative and pro-autophagic effect of N6L and point towards its possible use as adjuvant agent to the standard therapeutic protocols. Moreover, pseudopeptide N6L cross-linked to toxin saporin-S6n induced internalization of S6n inside glioblastoma cells in vitro. ${ }^{10}$ These results have shown that this conjugate induces cell death, with an $\mathrm{IC}_{50}$ four orders of magnitude lower than that, observed for free N6L. Furthermore, the preliminary in vivo study demonstrated efficiency in reducing the tumor mass in an orthotopic mouse model of glioblastoma. It appears that nucleolin, a molecule over-expressed on the surface of tumor cells, is an interesting therapeutic target. Several ligands, antagonists of nucleolin of various origins, such as AS1411, the F3 peptide and the multivalent pseudopeptide N6L have been developed and studied as perspective therapeutic tools against cancer. ${ }^{11}$ This study has revealed a selective apoptosis induced by two cationic peptides AM-2 and NC 783 in glioblastoma cell lines generated from primary tumors.

As a result of incubation with CPs $(2 \mu \mathrm{g} / \mathrm{ml})$, cell survival rate of the both glioblastoma lines significantly decreased compared to cells in control cells without the addition of CP: $5.1-7.1 \%$ vs $95-100 \%$ $(\mathrm{p}<0.05)$, Figure 1. Attention is drawn to similar cytotoxicities of CPs against both Glb-17 cells and Glb-Sh cell lines (Figure 1,2).

\section{Glb-Sh glioblastoma cell line viability}

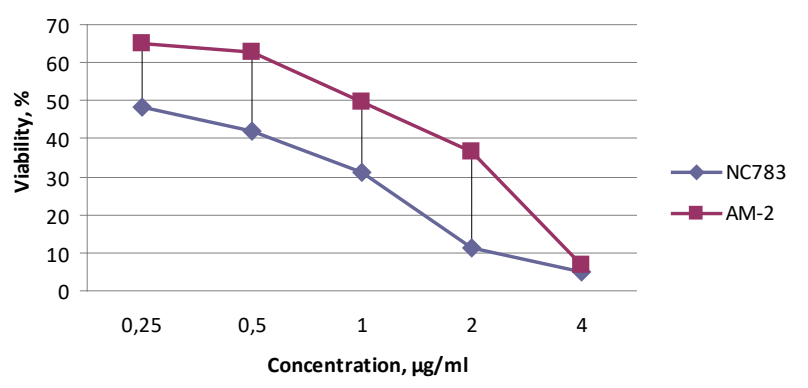

Figure I Results of MTT test using two $\mathrm{CPs}_{3} \mathrm{IC}_{50}=0.25 \mu \mathrm{g} / \mathrm{ml}$ for $\mathrm{NC} 783$ and l $\mu \mathrm{g} / \mathrm{ml}$-for AM-2.

\section{Glb-17 glioblastoma cell line viability}

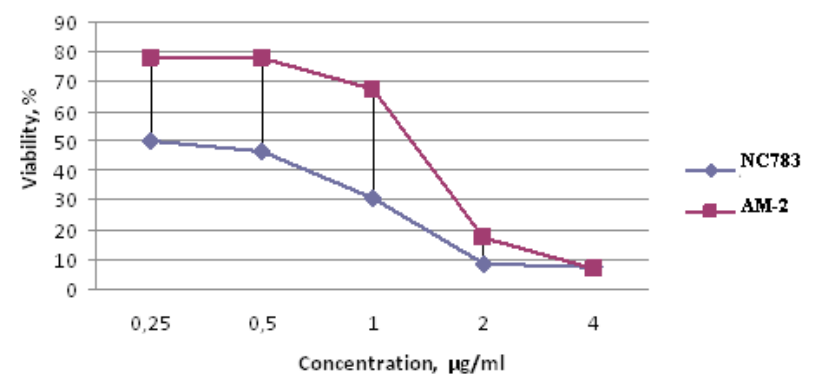

Figure 2 Results of MTT test using two CPs; for $\mathrm{NC783}, \mathrm{IC}_{50}=0.25 \mu \mathrm{g} / \mathrm{ml}$. for $\mathrm{AM}-2, \mathrm{IC}_{50}=1.25 \mu \mathrm{g} / \mathrm{ml}$.

As experiments have revealed, the survival rate of human skin fibrolblast H1036 line (control) after 3-days incubation with NC 783 or AM-2 did not significantly decrease (up to $92 \%$, not shown). These differences are likely related to the different expression levels of the surface nucleolin-the intended target for the peptides under study. To find the mechanisms of selective cytotoxicity of CPs, we have analyzed the expression of NCL and NPM in Glb-17 and Glb-Sh cell lines obtained by immunohistochemical staining with biotin-labeled monoclonal antibodies. Significant quantitative differences in the levels of proteins in glioblastoma and human fibroblasts have been revealed, Table 2 . The data indicate that NCL/NPM might consider as a tumor biomarkers.

Two hours after introduction of Cy5-NC811 $(1 \mu \mathrm{g} / \mathrm{ml})$ into microcells with Glb-17 and Glb-Sh cell cultures, passed over daily, apoptosis was observed, including chromatin fragmentation. It was confirmed by specific cell staining with Hoechst 33342, DAPI, PI, annexin-fluorescein (Ann-Cy3/6-CFDA), per 10-5 M/l. In addition, western blotting revealed the expression of $\mathrm{p} 53$ phosphorylated by Ser15, Figure 3. The activation of caspase 3 and 9 was also found by flow cytometry (not shown). These data indicate the induction of p53dependent apoptosis by cationic peptides. 
Table 2 Expression of NCL/NPM in glioblastoma and normal cells

\begin{tabular}{lccc}
\hline Parameter & Glb-Sh & Glb-I7 & HI036, control \\
\hline Relative density of biotin-labeled antibodies to NCL & $27.6 \pm 2.9$ & $21.3 \pm 1.6$ & $5.5 \pm 0.4$ \\
& & & $P<0.05$ \\
Relative density of biotin-labeled antibodies to NPM & $17.5 \pm 1.2$ & $16.7 \pm 1.1$ & $4.8 \pm 0.8$ \\
& & & $P<0.05$
\end{tabular}

It was also revealed (Figure 3A, B) that during the incubation of Glb-Sh cells, the level of free NCL decreases, the level of free NPM increases slightly, while the level of the p53 suppressor protein increases approximately twice. Changes in protein expression levels are associated with changes in transcription levels (Figure 3B), except $N P M$ gene. Really, the level of NPM mRNA decreases while free NPM level increases. It indicates the exit of nucleophosmin pentamer molecules from the nucleus into cytoplasm followed by degradation. The morphology of cells also corresponds to the beginning of apoptosis. Under phase contrast, there are many large chromatin globules on the periphery of the nuclei (Figure 3C). To confirm the role of CPs in the induction of glioblastoma cell apoptosis, the simulation of molecular interactions by pair docking was used (Figure 4,5).

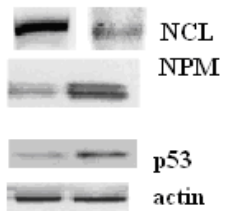

A

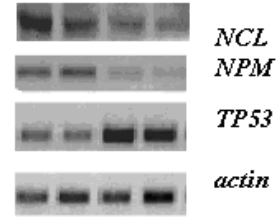

B

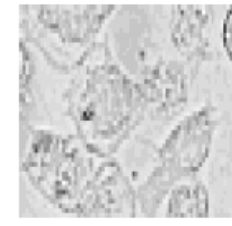

C
Figure 3 (A) Comparison of the results of western bloting and (B) RT PCR using mRNA from Glb-Sh glioblastoma cells: after I-2 (A) and I,2,3,4 hours (B) of cell incubation with CP AM-2. (C) Apoptosis in cell culture after 4 hours of incubation with CP AM-2. Chromatin fragmentation is visible, phase contrast $\times 400$.

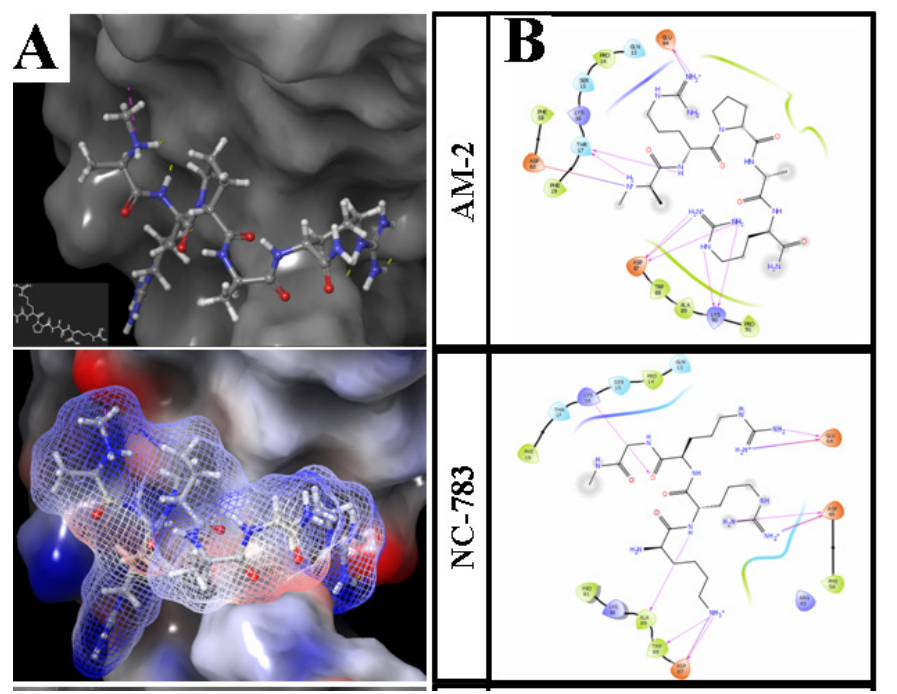

Figure 4 Docking results for PCs AM-2NCL. A. Upper: Positioned ligand AM-2 at NCL binding site. Equipotential surfaces of ligand (dashed) and protein (solid) are shown, red-negative, blue-positive, gray - neutral. B. Ligand interaction diagrams for two PCs. Docked free energy glide scores $=-6.26$ for AM-2; -6.53-for NC 783 .

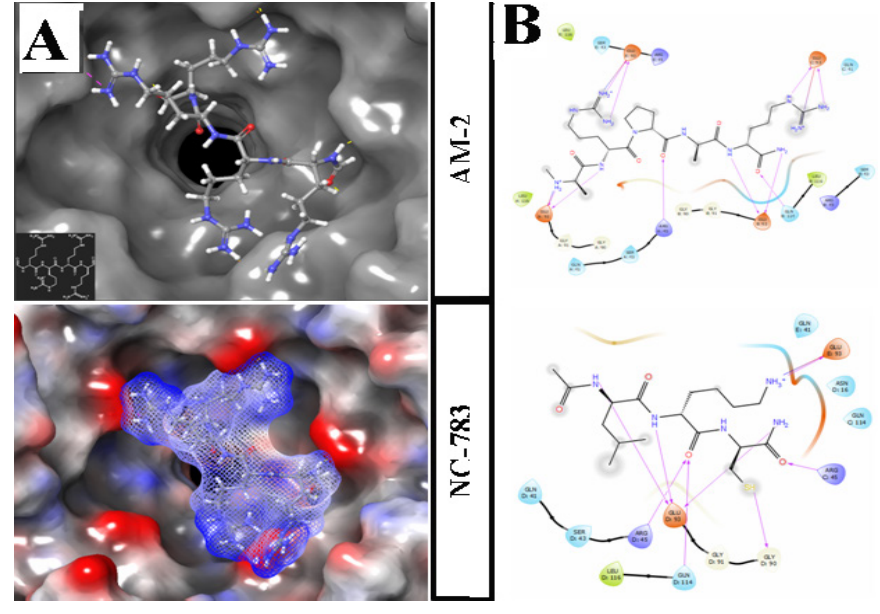

Figure 5 Docking results for NPMA. Upper: Positioned ligand (AM-2) in NPM binding site. Equipotential surfaces of ligand (dashed) and protein (solid), red negative, blue - positive, gray - neutral. B. Ligand interaction diagrams for two peptides. Docked free energy glide scores $=-6.45$ for AM-2; -6.33 for NC783, purple lines represents hydrogen bonds. Minimized structure with removed water and ions was performed by Maestro II. Docking box was put in the center of binding site of NPM upper site of a barrel, box length - 28 A.Force field - OPLS-3.

\section{Conclusion}

Human glioblactoma cell lines have shown high sensitivity to Arg/Lys-rich cationic peptides, induced selective apoptosis in tumor cells. Nucleolin/NCL and nucleophosmin/NPM are the most abundant nucleolar chaperone proteins, which are over-expressed in tumor cells. They are considered now as the cell proliferation markers and potential targets for cancer treatment. Simulation for two cationic peptides AM2, NC 783 and NCL/NPM interactions by molecular docking allows characterizing tested cationic peptides as ligands for these proteins. Binding between the peptides and cell surface nucleolin might trigger subsequent tumor cell apoptosis. The data obtained create the base for use the peptides as potent antitumor agents with high selective activity and low cytotoxicity.

\section{Acknowledgments}

None.

\section{Conflicts of interest}

The authors declare that there is no conflict of interest.

\section{References}

1. Lushnikova AA, Andreev SM, Kozhikhova KV, et al. Cationic peptides are the inducers of selective apoptosis in tumor cells. Allergy. 2018;73(S105):488 
2. Berger CM, Gaume X, Bouver P. The roles of nucleolin subcellular localization in cancer. Biochimie. 2015;113:78-85.

3. Lushnikova AA, Ponkratova DA, Kostarev AV, et al. Nucleolin and nucleophosmin as expected targets for cationic peptides, inducing tumor cell apoptosis. Journal of Bioinformatics and Genomics. 2018;3(8):1-8.

4. Hovanessian AG, Soundaramourty C, Khoury DE, et al. Surface expressed nucleolin is constantly induced in tumor cells to mediate calcum-dependent ligand internalization. PLoS One. 2010;5(12):e15787.

5. Lia W, Yao Z, Zhao I., et al. New perspectives of physiological and pathological functions of nucleolin (NCL). Life Sci. 2017;186:1-10.

6. Carvalho J, Paiva A, Campello MPC, et al. Aptamer-based targeted delivery of a G-quadruplex ligand in cervical cancer cells. Sci Rep. 2019;9(1):7945.

7. Fonseca NA, Rodrigues AS, Paulo RS, et al. Nucleolin overexpression in breast cancer cell sub-populations with different stem-like phenotype enables targeted intracellular delivery of synergistic drug combination. Biomaterials. 2015;69:76-88.
8. Lushnikova AA, Kostarev AV, Onyan AV, et al. Simulation between nucleolin and cationic peptides, inducing tumor cell apoptosis, by molecular docking. J Cancer Prev Curr Res. 2018;9(4):187-189.

9. Bendetti E, Antonosante A, Angelo MD, et al. Nucleolin antagonist triggers autophagic cell death in human glioblastoma primary cells and decreased in vivo tumor growth in orthotopic brain tumor model. Oncotarget. 2015;6(39):42091-42104.

10. Dhez A-Ch, Benedetti E, Antonosanta A, et al. Targeted therapy of human glioblastoma via delivery of a toxin through a peptide directed to cell surface nucleolin. J Cell Physiol. 2018;233(5):4091-4105.

11. Ferrara B, Belbekhouche S, Habert D, et al. Cell surface nucleolin as active bait for nanomedicine in cancer therapy: a promising option. Nanotechnology. 2021;32(32). 